\title{
海绵中提取的异臭椿萜类化合物作用靶标的识别
}

\author{
田 然 刘振明\$,*金宏威张亮仁林文翰” \\ (北京大学药学院, 天然药物及仿生药物国家重点实验室, 北京 100191)
}

\begin{abstract}
摘要: 采取了包括化学结构相似性学习、靶标聚类分析以及反向对接筛选等多种方法在内的综合性策略, 尝 试对中国南海海绵中提取得到的异臭椿萜类化合物进行生物学活性和作用靶标的预测. 结果表明: 这类化合物 具有治疗心肌缺血和抗肿瘤的潜在生物学活性; 表皮细胞生长因子受体(EGFR), 焦点(局部)粘着斑激酶 (FAK), 胰岛素样生长因子1受体 (IGF1-R), c-Src激酶以及血管表皮生长因子受体2 (VEGF-R2)是这类化合物 可能的作用靶标. IC 50 值从 $0.41 \mathrm{~g} \cdot \mathrm{m}^{-3}\left(0.41 \mu \mathrm{g} \cdot \mathrm{mL}^{-1}\right)$ 到 $9.8 \mathrm{~g} \cdot \mathrm{m}^{-3}\left(9.8 \mu \mathrm{g} \cdot \mathrm{mL}^{-1}\right)$ 不等. 活性数据显示这些海绵 提取的海洋天然产物可作为先导化合物, 通过进一步的优化获得新的药物. 同时还讨论了化合物与预测靶标的 结合模式, 结果显示四个化合物都与相应的受体有较好的结合.
\end{abstract}

关键词：海洋天然产物；异臭椿三萜类化合物；PASS 程序； 反向虚拟篮选；靶标识别；抗肿瘤活性 中图分类号: 0641

\section{Target Identification of Isomalabaricane Terpenes Extracted from Sponges}

\author{
TIAN Ran $\quad$ LIU Zhen-Ming ${ }^{\S, *} \quad$ JIN Hong-Wei ZHANG Liang-Ren ～LIN Wen-Han* \\ (State Key Laboratory of Natural and Biomimetic Drugs, School of Pharmaceutical Sciences, Peking University, \\ Beijing 100191, P. R. China)
}

\begin{abstract}
A strategy combining structure alignment and comparation, target cluster, and invert-docking screening were undertaken to detect the potential bioactivities of several isomalabaricane triterpenoids that were extracted from sponge. The prediction results revealed that the chemicals underwent myocardial ischemia treatment and had antineoplastic bioactivities. Enzymatic bioassays showed that epidermal growth factor receptor (EGFR), focal adhesion kinase (FAK), insulin-like growth factor 1 receptor (IGF1-R), c-Src kinase, and vascular endothelial growth factor receptor 2 (VEGF-R2) were potential targets for these compounds. They had $I_{50}$ values ranging from $0.41 \mathrm{~g} \cdot \mathrm{m}^{-3}\left(0.41 \mu \mathrm{g} \cdot \mathrm{mL}^{-1}\right)$ to $9.8 \mathrm{~g} \cdot \mathrm{m}^{-3}$ $\left(9.8 \mu \mathrm{g} \cdot \mathrm{mL}^{-1}\right)$, which is meaningful for the use of these marine natural products as leads for further modifications to new agents. Ligand-target binding mode with these compounds were undertaken and indicated that the four studied chemicals bound well with the targets.
\end{abstract}

Key Words: Marine natural product; Isomalabaricane triterpenoid; Program of Prediction of Activity Spectra for Substances; Reverse virtual screening; Target identification; Antineoplastic bioactivity

\footnotetext{
Received: November 22, 2010; Revised: February 17, 2011; Published on Web: April 13, 2011.

"Corresponding authors. LIU Zhen-Ming, Email: zmliu@bjmu.edu.cn; Tel: +86-10-82805514. LIN Wen-Han, Email: whlin@bjmu.edu.cn; Tel: +86-10-82806188.

${ }^{\S}$ These authors contributed equally to this work.

The project was supported by the Major National Science and Technology Program-Key Drug Scheme Funds (2009ZX09501-002) and National Natural Science Foundation of China (20802006).

重大新药创制国家科技重大专项(2009ZX09501-002)和国家自然科学基金 (20802006)资助项目
}

(C) Editorial office of Acta Physico-Chimica Sinica 


\section{1 引言}

从远古时代起, 人类就意识到可以从自然界中 寻找药物. 直到现在, 市场销售最好的药物中, 约有 三分之一都是天然产物, 或者是以天然产物为先导 结构而改造得到的. ${ }^{1,2}$ 现阶段, 我们用于新药来源的 天然产物通常都是来自于陆生生物. 但是, 伴随着陆 生物种研究的深入和枯竭, 新药的天然来源将会变 得越来越有限.

海洋占据了地球超过 $70 \%$ 的表面积, 大约存在 着 5 亿种的不同生物, 约为全球生物物种的一半, 拥 有的生物多样性远远超过想象. 海洋环境具有高盐、 高压、缺氧和避光的特性, 从而导致海洋生物在生活 习性、次级代谢产物的生物合成途径及酶催化反应 机制上与陆生生物完全不同, 可以提供全新的化学 结构. ${ }^{3-5}$ 目前, 寻找高效的、具有特异性的药物用于 人类疾病的治疗已经成为一个重要的趋势. 海洋生 物蕴藏着大量天然产物和新型化学实体, 越来越受 到研究者的关注. ${ }^{6-9}$

目前, 阻止海洋天然产物成为治疗药物的重要 原因是它们本身的结构复杂性以及作用靶标与药理 学机制不明确; 因此研究者无法依据靶标对它们及 其衍生物进行结构设计和化学修饰改造. 由于进行 蛋白质和基因水平上的生物活性篎选范围太广, 周 期较长, 耗费资金多, 目前大多数的海洋天然产物所 测定的初步生物活性是基于细胞水平的, 因此无法 知道其在生物体内作用的具体靶点. ${ }^{10,11}$ 虽然在此基 础上可以将具有较好生物活性的海洋天然产物作为 先导化合物, 通过合成和改变其不同取代基团来研 究其结构与活性的关系, 从而找出活性更好的结构 类型. 但是由于海洋天然产物结构独特, 其合成和改 造一般较难完成. ${ }^{12-14}$
本研究建立了一种基于化学结构相似性学习、 聚类分析以及反向对接找靶等多种方法在内的综合 性生物活性预测策略, 对从中国南海海绵中提取得 到的四个全新异臭椿萜类化合物(如图 1 所示)进行 生物学活性和作用靶标的预测. 这些化合物代表了 典型的异臭椿萜类化合物的结构特征.

\section{2 方法和材料}

本课题研究所用的异臭椿萜类化合物采自海南 岛三亚附近海域的海绵(Jaspis sp.)和采自中国南海 海域黄海绵(Rhabdastrella aff. Distincta) 中提取分离 所得, ${ }^{15,16}$ 具体化学结构如图 1 所示.

针对所选取化合物的靶标搜寻过程与策略如 图 2 所示. 具体来讲, 首先选择所研究异臭椿萜类 化合物的二维结构作为提问分子, 通过搜索 PASS (Prediction of Activity Spectra for Substances)系统所 提供的 SAR (Structure-Activities Relationship)训练 集合, 从分子骨架片段相似性出发进行初步生物学 活性的预测; 通过对所得到生物活性的聚类分析, 发现这些化合物的潜在生物活性. ${ }^{17,18}$ 进一步的研 究工作采用反向虚拟找靶方法, 对接实验室建立的 人类疾病相关蛋白结构信息数据库 (HDRP-SID, unpublished work), 对这些化合物的潜在靶标进行 精确预测, 并通过酶活性试验进行证实. 最后, 通过 对这些化合物与靶标激酶的结合模式研究进一步阐 述相互作用的空间结构特征, 为这类异臭椿萜类化 合物的进一步修饰和研究提供指导和依据.

\section{1 化合物结构的构建}

所有异臭椿萜类化合物的二维结构是在 ChemDraw Ultra 8.0 软件(http://www.chemcad.com) ${ }^{19}$ 中构 建的, 所有结构皆以 MOL 文件格式储存以备下一步
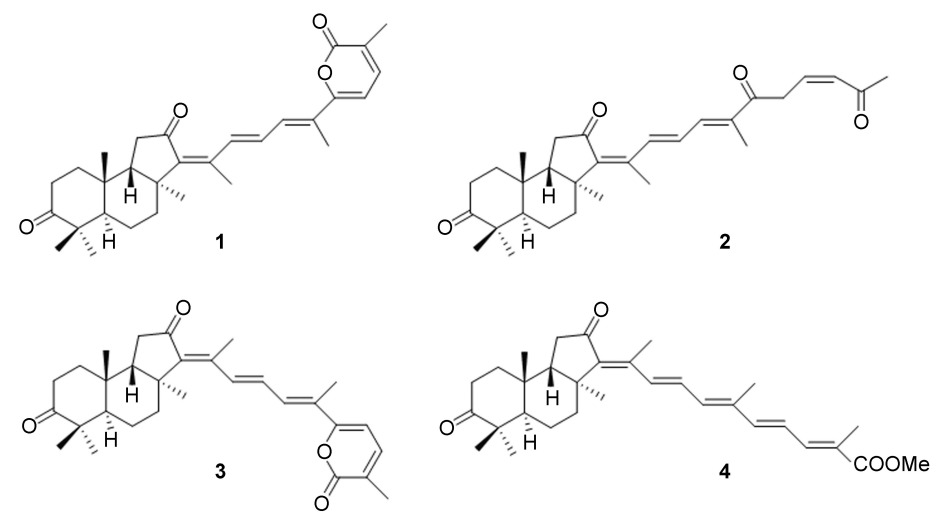

图 1 从 Jaspis sp. 和 Rhabdastrella aff. Distincta 中提取的 4 种异臭椿萜类化合物的结构式

Fig.1 Chemical structures of four isomalabaricane triterpenoids extracted from Jaspis sp. and Rhabdastrella aff. Distincta 
使用.

异臭椿萜类化合物的三维结构是在 SYBYL 6.91 软件包 ${ }^{20}$ 中构建的. 在优化过程中, 采用 Tripos 力场, 加载 Gasteiger 电荷, 先以共轭梯度法优化 1000 步, 再以最陡下降法优化至收玫, 收玫标准为 能量梯度 $\left(0.042 \mathrm{~kJ} \cdot \mathrm{mol}^{-1} \cdot \mathrm{nm}^{-1}\right)$. 所得结构均以 MOL2 文件格式存储以备下一步使用.

\section{2 基于小分子化合物结构的生物活性预测}

PASS 系统是基于小分子活性片段进行活性预 测的在线服务系统, ${ }^{21}$ 可以为类药化合物预测潜在的 生物活性. 该系统囊括了 35000 个以上的具有不同 生物活性的非同类化合物以及 500 种不同类型生物 活性, 包括药理学作用、作用机理、致突变性、致癌 性、致畸性和胚胎毒性等, 并由这些化合物构成了庞 大的训练集数据库. 该系统对于化合物生物活性的 预测正是基于对训练集的结构-生物活性关系的分 析得到的结果.

将已构建好的异臭椿萜类化合物的二维 MOL 格式结构输入 PASS 的 SAR 训练集数据库中. 经过 库搜索和结构匹配, 选取预测准确性 $\mathrm{Pa}>0.7$ 的部分 作为所研究化合物可能具有的生物活性, 并作为进 一步研究和分析的依据. 计算过程中的所有参数均 采用系统默认设置.
PASS 的基本元素包括生物活性表述、化学结构 描述、化合物训练集、训练程序和预测程序. 训练程 序生成各类型生物活性的结构-生物活性关系, 当 输入提问化合物二维结构时, 程序会自动将该结构 以 MNA (Multilevel Neighborhoods of Atoms)描述符 方式表示, 然后预测程序将提问化合物与训练集相 匹配, 从而得到提问化合物可能的生物活性.

\section{3 基于蛋白质三维结构的靶标搜寻的反向分 子对接}

三维结构的靶标搜寻方法需要具备三个必要元 素: 靶标三维结构数据库、反向分子对接程序以及 快速可靠的打分函数.

\subsection{1 靶标三维结构数据库}

选取了本实验室开发的人类疾病相关蛋白结构 信息数据库 HDRP-SID 作为异臭椿萜类化合物的靶 标数据库篮选对象, 通过对生物活性预测所得到的 疾病相关靶标的反向对接精确确证化合物在生物体 内的潜在作用靶点.

人类疾病相关蛋白结构信息数据库收录了 2266 套蛋白三维结构与结合位点数据, 包括约 600 个药物靶点以及超过 1700 个与疾病相关的蛋白质, 与人类血液循环、免疫系统、内分泌系统、呼吸系统、 遗传紊乱、肿瘤、细菌感染、传染病及寄生疾病等九

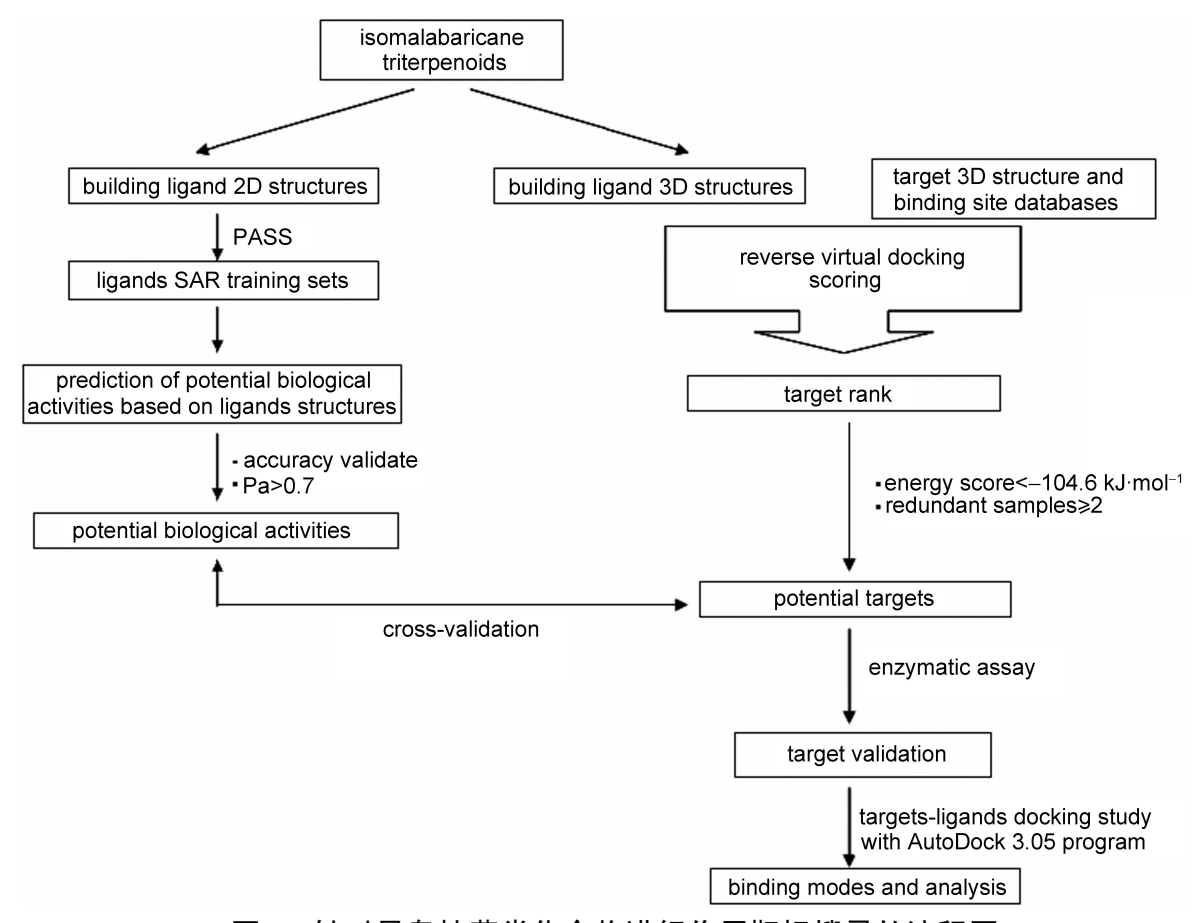

图 2 针对异臭椿萜类化合物进行作用靶标搜寻的流程图

Fig.2 Flow chart for target identification and biological testing of isomalabaricane terpenes extracted from sponges of the South China Sea 
大类疾病相关. 对于同一种蛋白, PDB 数据库 (http:// www.rcsb.org)中往往有许多来源不同、分辨率不同、 结构状态不同的三维结构, 该数据库尽量收集了 PDB 中所有的典型结构. 由于结合不同抑制剂时, 蛋白的三维结构尤其是结合口袋处的结构会发生变 化. 对于此种情况, 数据库收录了至少两套符合以上 挑选条件的结合了不同抑制剂的蛋白结构, 作为这 些蛋白的圥余结构, 以保证在篎选时可以给予这些 重要蛋白充分的考虑.

\subsection{2 分子对接程序}

基于蛋白质三维结构的反向靶标搜寻策略是建 立在 DOCK 4.06 程序 ${ }^{22}$ 之上的一种改进. 虽然 DOCK 4.06程序本身的计算精确度不如已知的其他 很多分子对接程序, ${ }^{23-27}$ 但它所具有的计算速度快, 通过简单修饰就可以实现配体库对靶标库的对接策 略等优点使得它可以很方便地被用于配体的反向靶 标库对接和生物活性的预测研究中.

对靶标三维结构的前处理及分子对接过程中参 数设置如下: 除去原靶标晶体结构中的水分子、复 合物配体分子及辅助因子, 保留金属离子, 以 PDB 文件格式保存. 在前面操作的基础上添加全氢原子, 并在 AMBER7 FF99 力场上加载电荷, 以 MOL2 文 件格式保存. 采用 SPHGEN程序, 选取原靶标结构 中的复合物配体或已证实的关键氨基酸残基周围 $0.6 \mathrm{~nm}$ 范围内的氨基酸残基作为每个靶标的活性位 点并生成相应的负像.

进行分子对接时, atom_model 参数设置为 united atom models, 每个靶标与每个异臭椿萜类化合物最 后保存的结合模式个数设置为 1 , 即只保存打分最 高、认为结合最好最合理的模式. 其它参数均采用缺 省值. 通过 perl 脚本程序实现了 DOCK 4.06 程序对 多蛋白结构数据库结构的调用, 所得的反向虚拟篮 选结果依照能量打分由低到高的顺序排列, 保留能 量低于 $-104.6 \mathrm{~kJ} \cdot \mathrm{mol}^{-1}$ 的靶标作进一步分析. 将最 后得到的结合能力评价结果按照受体结合口袋与配 体的分子大小进行权重处理后进行输出, 从而实现 了多重对接策略下的评价结果的可比性与真实性.

\subsection{3 打分函数}

这里选取 DOCK4.06程序所提供的能量打分作 为一次打分函数, 并按照蛋白靶标的结合口袋大小 和静电的数目对所得的打分结果进行权重修正和二 次排名.

\section{4 酶抑制实验}

根据反向靶标搜寻结果, 对所研究的异臭椿萜 类化合物与预测所得到的表皮细胞生长因子受体, 焦点(局部)粘着斑激酶, 胰岛素样生长因子 1 受体, $\mathrm{c}-\mathrm{Src}$ 激酶以及血管表皮生长因子受体 2 等部分蛋白 激酶的抑制活性进行了酶学研究和测定.

所有蛋白激酶的活性均通过测定转移到激酶底 物上的 $\left[\gamma-{ }^{33} \mathrm{P}\right]$-ATP 的 $\left[{ }^{33} \mathrm{P}\right]$ 放射活度来检测, 所有操作 均在 96 孔板中进行. 反应混合液包含: (1) $2 \times 10^{-8}$ $\mathrm{m}^{-3}(20 \mu \mathrm{L})$ 酶反应缓冲液, 包括 $60 \mathrm{mmol} \cdot \mathrm{L}^{-1}$ 的 HEPES (4-(2-hydroxyethyl)-1-piperazineethanesulfonic) $-\mathrm{NaOH}(\mathrm{pH}=7.5), 3 \mathrm{mmol} \cdot \mathrm{L}^{-1} \mathrm{MgCl}_{2}, 3 \mathrm{mmol} \cdot \mathrm{L}^{-1}$ 的 $\mathrm{MnCl}_{2}, 3 \mu \mathrm{mol} \cdot \mathrm{L}^{-1}$ 的 $\mathrm{Na}_{3} \mathrm{VO}_{4}, 1.2 \mathrm{mmol} \cdot \mathrm{L}^{-1} \mathrm{DTT}$ (dithiothreitol), $50 \mathrm{~g} \cdot \mathrm{m}^{-3}\left(50 \mu \mathrm{g} \cdot \mathrm{mL}^{-1}\right) \mathrm{PEG}_{20000}, 1$ $\mu \mathrm{mol} \cdot \mathrm{L}^{-1}\left[\gamma^{-33} \mathrm{P}\right]$-ATP (adenosine triphosphate)(PerkinElmer, Inc. Waltham, MA 02451, USA. 每孔约 $5 \times$ $\left.10^{5} \mathrm{~min}^{-1}\right)$; (2) $5 \times 10^{-9} \mathrm{~m}^{-3}(5 \mu \mathrm{L}) \mathrm{ATP}$ 水溶液; (3) $5 \times$ $10^{-9} \mathrm{~m}^{-3}(5 \mu \mathrm{L}$ ) 测试化合物的 DMSO (dimethylsulfoxide)溶液; (4) $1 \times 10^{-8} \mathrm{~m}^{-3}(10 \mu \mathrm{L})$ 多肽底物 Poly (Glu, Tyr $)_{4: 1}$ (Cell Signaling Technology, Inc. 2 Trask Lane, Danvers, MA 01923); (5) $1 \times 10^{-8} \mathrm{~m}^{-3}(10 \mu \mathrm{L})$ 纯净的重 组蛋白激酶(Bio-WORLD, Dublin, USA), 酶反应液 总体积为 $5 \times 10^{-8} \mathrm{~m}^{-3}(50 \mu \mathrm{L})$.

加入 $1 \times 10^{-8} \mathrm{~m}^{-3}(10 \mu \mathrm{L})$ 重组蛋白激酶启动酶反 应, $30{ }^{\circ} \mathrm{C}$ 水浴孵育 $80 \mathrm{~min}$. 加入 $5 \times 10^{-8} \mathrm{~m}^{-3}(50 \mu \mathrm{L})$ 的 $2 \%(V / V) \mathrm{H}_{3} \mathrm{PO}_{4}$ 溶液结束反应, 用 $2 \times 10^{-7} \mathrm{~m}^{-3}(200$ $\mu \mathrm{L})$ 的 $0.009 \mathrm{~g} \cdot \mathrm{mL}^{-1} \mathrm{NaCl}$ 溶液冲洗 96 孔板两次, 采 用微量闪㷧计数器测定其放射性, 以所测放射性计 数率来反映激酶活性. 除了特殊说明外, 所有试剂均 为来源于 Sigma-Aldrich 北京公司的分析纯试剂.

\section{5 异臭椿萜类化合物与确证靶标的结合模式分析}

选用 AutoDOCK 3.05 程序, ${ }^{28,29}$ 对确证靶标分子 与异臭椿萜类化合物的结合模式进行分析研究.

对接过程所需要的受体三维结构均来自 PDB 结构数据库, 包括 EGF-R(PDB ID: 1XKK), FAK (PDB ID: 2ETM), IGF1-R(PDB ID: 2OJ9), c-Src 激酶 (PDB ID: 1NZL)和 VEGF-R2(PDB ID: 1Y6B).

将 PDB 结构文件中的水分子、离子、配体小分 子、辅助因子及多余的蛋白质去除, 仅保留与结合模 式研究有关的蛋白质单体结构, 并修补各蛋白质单 体结构中缺失的氨基酸残基.

使用 AutoDock Tools 为受体和配体添加氢原子 及电荷, 并为受体分子添加溶剂化参数. 本研究课题 中, 受体分子加载的是 Kollman 电荷, 配体分子添加 
的是 Gasteiger 电荷. 对接过程中所做格点的范围 是以原 PDB 文件中复合物配体分子为中心, 在 $x$ 、 $y 、 z$ 轴上分别延伸 50 个格点的范围. 对接采用的是 GALS (Lamarckian Genetic Algorithm) 算法, 对每一 个配体进行 100 次单独的运算. 对接构象的聚类分 析采用的 RMSD 值是 $0.1 \mathrm{~nm}$. 其余参数均采用默 认值.

\section{3 结果与讨论}

\section{1 基于 PASS 程序的生物活性实验预测}

所选取化合物基于配体结构片段的生物活性预 测结果如表 1 所示: $\mathrm{Pa}$ 为具有对应生物学活性的概 率; $\mathrm{Pi}$ 为非生物学活性的概率. 由以上结果可以看 出, 由于是同一类型的化合物, 且结构差异不是特别 大, PASS 预测得到的结果也十分相近. 尤其是 $\mathbf{1}$ 和 $\mathbf{3}$ 这一对化合物, 是 C13、C14 间双键的顺反异构体, 异构体彼此之间的预测结果是完全相同的. 由于 PASS 程序考虑的只是小分子化合物的二维结构, 而 且是以 MNA 的方式将每个化合物拆分成描述符来 表示, 双键的顺反异构体拆分的描述符是一致的, 因 此所得的预测结果也是完全相同的. 这个结果一方 面说明了 PASS 程序在光学异构体活性预测方面的 不足和缺陷, 但另一方面也可以体现了预测结果的 准确性与可信性.

上述结果中引起我们注意的主要有两点:

(1) 所有化合物预测结果中都包含有抗肿瘤剂 的生物活性; (2) 含有内酯环的化合物具有心肌缺血. 治疗的生物活性.

第一点说明此类化合物极有可能具有抗肿瘤的 生物活性, 这方面通过文献和抗肿瘤细胞活性实验 得到了证实. 结果表明: (1) 侧链结构中的六元不饱 和内酯部分是最重要的影响因素, 最典型的表现就 是在抑制 HL-60人白血病细胞增殖的试验中, 若无 六元不饱和内酯环, 其抗肿瘤活性会有很大程度的 下降; (2) C-13/C-14 烯双键的构型, C-13/C-14 位为 Z-式构型样品的活性要低于 E-式构型; (3) C-3 位取 代基中对肿瘤细胞增殖的抑制率大小依次为羟基 $>$ 羰基>乙酰氧基, 但是相对来说对抗肿瘤活性的影 响不如以上两种因素那么大.

针对第二点预测结果, 我们最初怀疑内酯环为 这一类活性的关键官能团, 所以又以内酯环结构作 为提问结构进行 PASS 预测, 但所得心肌缺血治疗 项的 $\mathrm{Pa}$ 值只有 0.714 . 我们尝试以图 3 中所示 $\mathrm{a}$ 结构
作为提问结构进行预测, 所得心肌缺血治疗项的 $\mathrm{Pa}$ 高达 0.988. 我们以此结构在 MDL 数据库中进行搜 索, 期望可以找到与 $\mathrm{a}$ 结构类似或含有此结构的已 知具有该生物活性的化合物, 但结果并不理想. 随 后, 我们采用 $\mathrm{b}$ 结构进行 MDL数据库搜索, 得到如 $\mathrm{c}$ 所示的化合物, 它具有抗心绞痛作用, 从而说明异臭 椿萜类化合物所包含的部分骨架片段可能具有心肌 缺血治疗作用.

\section{2 基于蛋白质三维结构的靶标搜寻}

PASS 活性预测结果和细胞实验表明, 异臭椿萜 类化合物对多种肿瘤细胞具有良好的抑制活性, 备 受科学家们的关注, 有望成为一类新型、高效、低毒 的抗肿瘤制剂. 同时, 异臭椿萜类化合物所包含的部 分骨架片段可能具有心肌缺血治疗作用的预测, 使

表 1 基于配体结构片段的生物活性预测

Table 1 Predicted results of biological activities based on substrate fragments

\begin{tabular}{|c|c|c|c|}
\hline Compound & $\mathrm{Pa}$ & $\mathrm{Pi}$ & Biological activity \\
\hline \multirow{7}{*}{1} & 0.954 & 0.005 & myocardial ischemia treatment \\
\hline & 0.844 & 0.007 & antineoplastic \\
\hline & 0.756 & 0.063 & phosphatase inhibitor \\
\hline & 0.740 & 0.012 & carminative \\
\hline & 0.734 & 0.030 & cholesterol synthesis inhibitor \\
\hline & 0.731 & 0.016 & apoptosis agonist \\
\hline & 0.707 & 0.043 & cardiovascular analeptic \\
\hline \multirow[t]{8}{*}{2} & 0.857 & 0.020 & phosphatase inhibitor \\
\hline & 0.829 & 0.054 & antiseborrheic \\
\hline & 0.824 & 0.007 & apoptosis agonist \\
\hline & 0.818 & 0.034 & mucomembranous protector \\
\hline & 0.767 & 0.011 & antineoplastic \\
\hline & 0.740 & 0.047 & B-adrenergic-receptor kinase inhibitor \\
\hline & 0.722 & 0.007 & dermatologic \\
\hline & 0.715 & 0.019 & oxidoreductase inhibitor \\
\hline \multirow[t]{7}{*}{3} & 0.954 & 0.005 & myocardial ischemia treatment \\
\hline & 0.844 & 0.007 & antineoplastic \\
\hline & 0.756 & 0.063 & phosphatase inhibitor \\
\hline & 0.740 & 0.012 & carminative \\
\hline & 0.734 & 0.030 & cholesterol synthesis inhibitor \\
\hline & 0.731 & 0.016 & apoptosis agonist \\
\hline & 0.707 & 0.043 & cardiovascular analeptic \\
\hline \multirow[t]{7}{*}{4} & 0.871 & 0.021 & mucomembranous protector \\
\hline & 0.846 & 0.023 & phosphatase inhibitor \\
\hline & 0.823 & 0.058 & antiseborrheic \\
\hline & 0.822 & 0.007 & apoptosis agonist \\
\hline & 0.789 & 0.028 & $\begin{array}{l}\text { aminocarboxymuconate-semialdehyde } \\
\text { decarboxylase inhibitor }\end{array}$ \\
\hline & 0.730 & 0.014 & antineoplastic \\
\hline & 0.726 & 0.007 & dermatologic \\
\hline
\end{tabular}

The predicted activity spectrum is presented in PASS by the list of activities with probabilities "to be active" (Pa) and "to be inactive" (Pi) calculated for each activity. 
<smiles>C/C=C/C=C(\C)c1ccc(C)c(=O)o1</smiles>

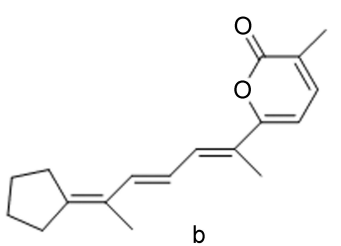<smiles>COc1cc(/C(C)=C\C=C\C=C\C=C\c2[nH]ccc2Cl)oc(=O)c1C</smiles>

图 3 具有心肌缺血治疗活性结构片段的搜寻与预测

Fig.3 Searching for the chemical fragment related to myocardial ischemia treatment

得找到并确定此类化合物的作用靶标对于进一步的 药学研究和应用具有重要意义.

由于每个化合物的反向对接预测结果都比较 多, 靶标数目往往超过 100 个. 它们中间有些功能 已明确, 与多种疾病相关, 有些功能至今还是未知 的. 同时, 在分子对接过程中还会存在不少假阳性
的情况. 基于以上两点原因, 为了提高结果的可信 度和减少假阳性情况的发生概率, 我们从初步篎选 排名结果中再选择出现频率在两次以上的靶标作 为最终结果如表 2 所示. 从表 2 中可以看到: 有些 蛋白质靶标在四个化合物的篎选最终结果中都有 出现, 有些靶标出现在两个化合物的篎选最终结果

Table 2 List of putative protein targets from reverse docking with HDRP-SID database

\begin{tabular}{|c|c|c|}
\hline Compound & Putative protein target & Related disease \\
\hline 1 & beta-glucosidase & group B streptococcal pneumonia \\
\hline 1 & carbonic anhydrase, type IV, II & $\mathrm{n} / \mathrm{a}$ \\
\hline 2 & serine proteinase alpha-thrombin & haemostatic disorders \\
\hline 2 & dihydrofolate reductase & $\begin{array}{l}\text { autoimmune diseases, bacterial infections, cancers, chagas ' disease, fungal } \\
\text { diseases, inflammation, leishmania infections, malaria, microbial Infections, } \\
\text { P. carinii pneumonia, parasitic diseases, toxoplasma infections, tuberculosis }\end{array}$ \\
\hline 2 & neuraminidase & influenza, virus not identified \\
\hline 3 & beta-glucosidase & group B streptococcal pneumonia \\
\hline 3 & $\mathrm{Fab}^{\prime}$ fragment of monoclonal antibody $\mathrm{Db} 3$ & $\mathrm{n} / \mathrm{a}$ \\
\hline 3 & human neutrophil collagenase & tumor invasion, joint destruction \\
\hline 3 & tyrosine-protein kinase transforming protein & \\
\hline & $\mathrm{SRC}$ & \\
\hline 4 & insulin receptor & diabetes mellitus \\
\hline 4 & peroxidase & $\mathrm{n} / \mathrm{a}$ \\
\hline 4 & atrial natriuretic peptide receptor A & $\mathrm{n} / \mathrm{a}$ \\
\hline 1,2 & aldehyde reductase & $\begin{array}{l}\text { diabetic neuropathy, diabetic retinopathy, neuropathic pain, } \\
\text { noninsulin-dependent diabetes mellitus }\end{array}$ \\
\hline 1,3 & human fibroblast collagenase & emphysema, chondrosarcoma \\
\hline 1,4 & memapsin 2 (beta-secretase) & alzheimer's disease \\
\hline 2,3 & D-xylose Isomerase & $\mathrm{n} / \mathrm{a}$ \\
\hline 2,3 & alcohol dehydrogenase & $\mathrm{n} / \mathrm{a}$ \\
\hline 2,4 & HIV protease & \\
\hline 2,4 & thymidylate synthase & $\begin{array}{l}\text { breast cancer; colorectal cancer; fungal diseases; gastric cancer; hepatocellular } \\
\text { carcinoma; ovarian cancer; pancreatic cancer; proliferative diseases }\end{array}$ \\
\hline 3,4 & nitric oxide synthase & $\begin{array}{l}\text { cardiovascular disease, colon cancer, helminth infection, inflammation, } \\
\text { schizophrenia, sepsis }\end{array}$ \\
\hline 3,4 & thiamin pyrophosphokinase & $\mathrm{n} / \mathrm{a}$ \\
\hline $1,2,3,4$ & penicillopepsin & $\mathrm{n} / \mathrm{a}$ \\
\hline $1,2,3,4$ & epidermal growth factor receptor & \\
\hline $1,2,3,4$ & casein kinase II (protein kinase CK2) & breast cancer, cancers \\
\hline $1,2,3,4$ & vascular endothelial growth factor receptor 2 & \\
\hline $1,2,3,4$ & endothiapepsin & $\mathrm{n} / \mathrm{a}$ \\
\hline $1,2,3,4$ & thermolysin & $\mathrm{n} / \mathrm{a}$ \\
\hline $1,2,3,4$ & focal adhesion kinase 1 & \\
\hline $1,2,3,4$ & insulin-like growth factor I receptor & \\
\hline
\end{tabular}


中, 有些化合物虽然只出现在一个化合物的管选最 终结果中, 但它在其它化合物的篎选初步结果中也 曾出现了一次.

\section{3 酶抑制实验}

由于异臭椿萜类化合物的样品量限制, 我们测 定了所研究化合物对表皮细胞生长因子受体、焦点 (局部) 粘着斑激酶, 胰岛素样生长因子 1 受体, c-Src 激酶以及血管表皮生长因子受体 2 等部分蛋 白激酶的生物活性. 所有蛋白激酶的活性均通过测 定转移到激酶底物上的 $\left[\gamma-{ }^{33} \mathrm{P}\right]-\mathrm{ATP}$ 的 $\left[{ }^{33} \mathrm{P}\right]$ 放射活度 来检测. 活性测定结果如图 4 所示.

从酶抑制实验结果可以看出, 各化合物确实可 以与这些激酶相结合, 抑制其酶活性, 说明 EGFR, FAK, IGF1-R, c-Src 激酶和 VEGF-R2 是这几个化合 物甚至是异臭椿萜类化合物的潜在作用靶标. 上述 酶活性实验结果表明, 这些化合物对 IGF1-R 和 c$\mathrm{Src}$ 激酶的抑制活性最好, 对 FAK 相对较差; 化合 物 $\mathbf{2}$ 与其它三个相比活性稍弱; 化合物 $\mathbf{1}$ 和 $\mathbf{3}$ 为 C13、C14 间双键的顺反异构体, 但它们对各激酶的 抑制活性比较接近, 区别并不明显; 化合物 4 虽然 也不含有内酯环, 但其对各激酶的抑制活性比 2 要 好, 与含有内酯环的 $1 、 3$ 活性非常接近.

\section{4 结合模式分析}

我们对所研究化合物与上述五个蛋白激酶的空 间结合模式进行了分子识别研究, 选取了 EGF-R (PDB ID: 1XKK), FAK (PDB ID: 2ETM), IGF1-R (PDB ID: 2OJ9), c-Src 激酶(PDB ID: 1NZL), VEGFR2(PDB ID: 1Y6B)五个晶体结构作为相互作用的 研究对象. 对接结果如图 5 所示.

从图 5 中可以看出, 我们所研究的异臭椿萜类 化合物均深埋于上述激酶的结合口袋中, 大多数 可以与激酶形成氢键. 所有化合物以相似的结合 模式与激酶相互作用, 但由于 C13、C14 间双键的 构象不同, 相同构象的化合物在激酶口袋中采取 相同的延伸方向. 我们还发现, 所研究激酶活性口 袋的狭长孔道形状正适合于上述异臭椿萜类化合 物的长链结构. 由于上述化合物中仅含有少量的 极性基团, 在疏水接触与靶点激酶的相互作用中 起关键作用.

所研究的四个化合物以相似的模式与表皮生 长因子受体 EGFR 相结合, C13、C14 间双键构型相 同的化合物延伸方向相同. 化合物 1、2、3 皆可与铰 链区的 Cys797 上的 $\mathrm{N}$ 原子形成氢键. 化合物 1 还可

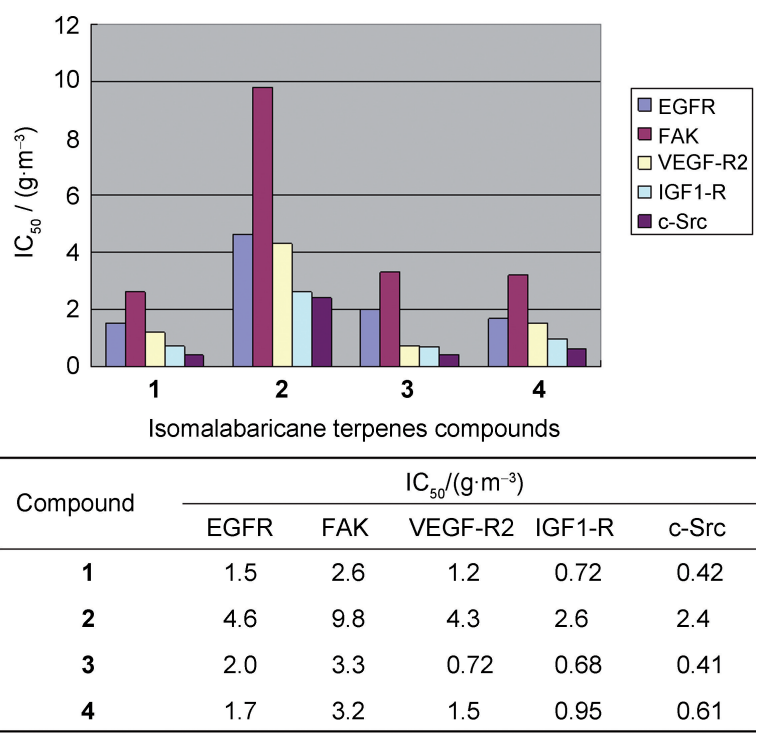

图 4 四个异臭椿萜类化合物对 EGFR、FAK、IGF1-R、 c-Src、VEGF-R2 生物活性 $\left(\mathrm{IC}_{50}\right)$ 的抑制

Fig.4 IC $\mathrm{I}_{50}$ values with the inhibition of EGFR, FAK, IGF1-R, c-Src, and VEGF-R2 by those four compounds

以与残基 $A s p 800$ 上的 $\mathrm{O}$ 原子之间形成氢键. 化合 物 4 由于较其它化合物支链较长, 仅能与 Tyr998 的 酚羟基形成氢键. 与 FAK 蛋白激酶的对接结果显 示, 化合物 1、2 中 A 环的羰基可与激酶的 Arg550上 的胍基 $\mathrm{N}$ 原子和 Ser568 的羟基形成氢键. 虽然其它 两个化合物由于构象不同未能与 FAK形成氢键, 但它们可与此激酶以疏水作用紧密结合.

从图 5 中我们可以清楚地看到: 除化合物 1 以 外, 其它三个化合物都可与表皮血管生长因子受体 VEGF-R2 铰链区 Lys866 的氨基 $\mathrm{N}$ 原子形成氢键. 构型相同的化合物在此激酶的结合口袋中采取相 同的方向延伸. 上述化合物与 IGF1-R 的相互作用 模式提供了相对更多的信息. 化合物 $1 、 2$ 都可与酶 上 Ser979 的羟基 $\mathrm{O}$ 和氨基 $\mathrm{N} 、 \mathrm{Phe} 980$ 氨基 $\mathrm{N}$ 原子形 成氢键; 化合物 3 可与激酶 Phe 980 氨基 $\mathrm{N}$ 和 Gly1055 氨基 $\mathrm{N}$ 原子形成氢键; 化合物 4 不仅可以 与激酶 Ser979 的羟基 $\mathrm{O}$ 形成氢键, 而且可以与 Phe980、Lys1003 和 Gly1055 的氨基 N 原子形成牢 固的氢键. 我们猜测 IGF1-R 与所研究化合物相互 作用的关键氨基酸残基为 Ser979 及 Phe 980 .

化合物 1 和 2 都可以与 c-Src 激酶的 Lys309 的 氨基 $\mathrm{N}$ 原子形成氢键; 化合物 $\mathbf{1}$ 和 $\mathbf{3}$ 都可以与激酶 $\operatorname{Arg} 261$ 的胍基 $\mathrm{N}$ 原子形成氢键; 所有化合物都可与 激酶 $\mathrm{Thr} 285$ 的羟基 $\mathrm{O}$ 原子形成氢键; 而化合物 4 又 可与 $\mathrm{Arg} 281$ 的胍基 $\mathrm{N}$ 原子形成氢键. 


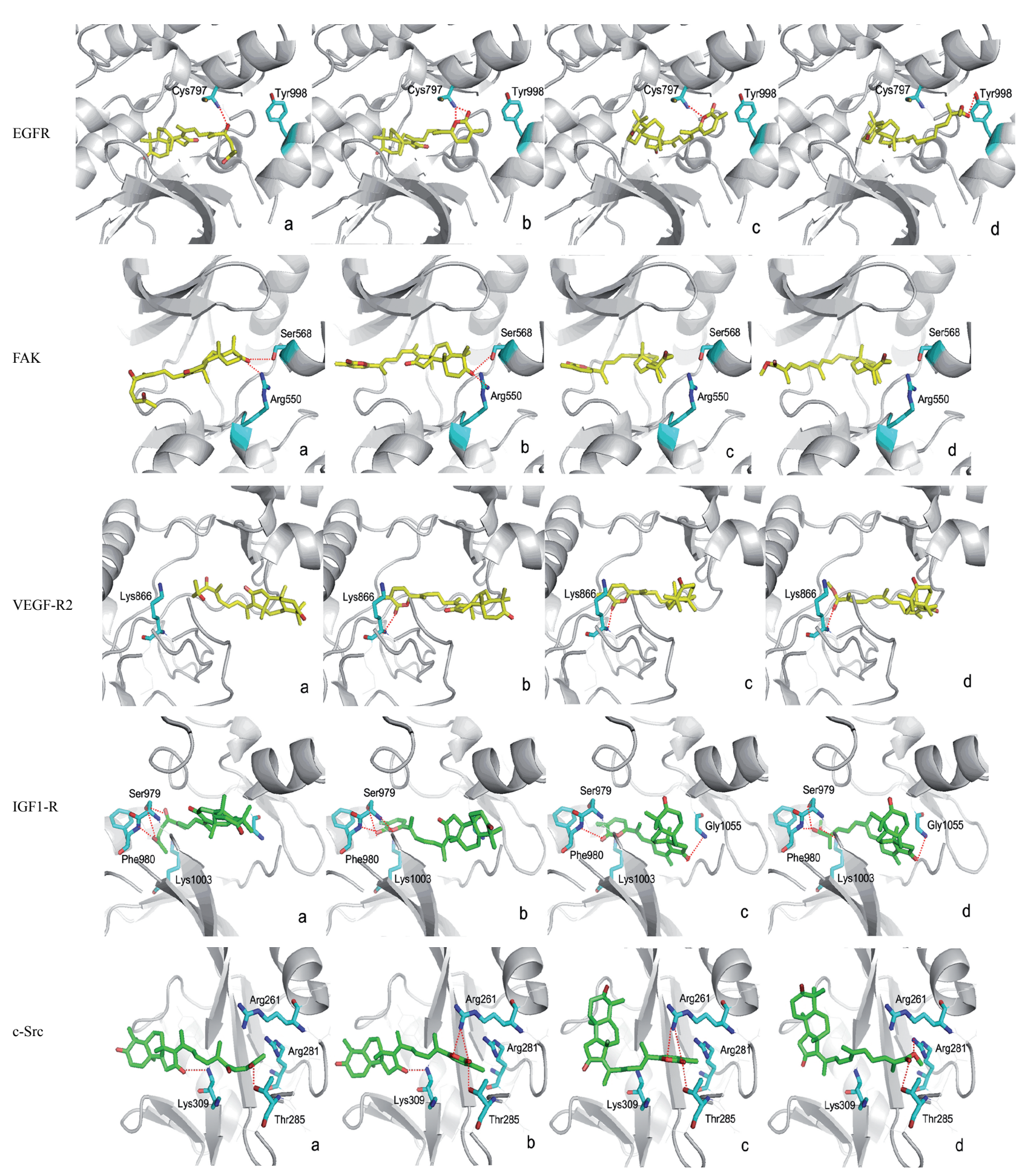

图 5 异臭椿萜类化合物与 EGFR, FAK, VEGF-R2, IGF1-R 以及 c-Src 激酶的相互作用模式

Fig.5 Binding of isomalabaricane terpenes with potential targets EGFR, FAK, VEGF-R2, IGF1-R and c-Src kinase The symbols a, b, c, $d$ in each figure represent compound $\mathbf{1 , 2}, \mathbf{3}, \mathbf{4}$, respectively.

\section{4 结 论}

本研究工作显示, 异臭椿萜类化合物可能具有 抗肿瘤的生物活性, 而且其中含有内酯环的化合物 可能具有心肌缺血治疗作用.

基于分子对接的反向虚拟筛选方法, 预测得到
了异臭椿萜类可能的作用靶标, 并用酶活性试验验 证了此类化合物确实可以抑制表皮细胞生长因子 受体、焦点(局部)粘着斑激酶、胰岛素样生长因子 1 受体、c-Src 激酶以及血管表皮生长因子受体 2 的酶 活性, 而且进一步研究和推测了它们的结合模式. 
这几种蛋白激酶均与肿瘤生成相关, 已经或可能成 为抗肿瘤靶标, 与前面 PASS 预测结果相互验证, 这 不仅证明了以上两种方法应用于海洋天然产物的 靶标预测具有一定的可行性和准确性, 更为异臭椿 萜类化合物的进一步研究提供了重要信息和理论 基础.

\section{References}

(1) Newman, D. J.; Cragg, G. M. J. Nat. Prod. 2007, 70, 461.

(2) Newman, D. J.; Cragg, G. M. Future Med. Chem. 2009, 1, 1415.

(3) Simmons, T. L.; Andrianasolo, E.; McPhail, K. Mol. Cancer Ther. 2005, 4, 333.

(4) Takamatsu, S.; Hodges, T. W.; Rajbhandari, I. J. Nat.Prod. 2003, 66, 605 .

(5) Paul, V. J.; Arthur, K. E.; Ritson-Williams, R. Biol. Bull. 2007, 213, 226.

(6) Nagle, D. G.; Zhou, Y. D. Phytochem. Rev. 2009, 8, 415.

(7) Villa, F. A.; Gerwick, L. Immunopharmacol. Immunotoxicol. 2010, 32, 228.

(8) Molinski, T. F.; Dalisay, D. S.; Lievens, S. L.; Saludes, J. P. Nat. Rev. Drug Discov. 2009, 8, 69.

(9) Bugni, T. S.; Richards, B.; Bhoite, L.; Cimbora, D.; Harper, M. K.; Ireland, C. M. J. Nat. Prod. 2008, 71, 1095.

(10) Thakur, N. L.; Jain, R.; Natalio, F.; Hamer, B.; Thakur, A. N.; Müller, W. E. Biotechnol. Adv. 2008, 26, 233.

(11) Faulkner, D. J. Nat. Prod. Rep. 2000, 17, 1.

(12) Nicolaou, K. C.; Vourloumis, D.; Winssinger, N. Angew Chem. Int. Edit. 2000, 39, 44.

(13) Yeung, K. S.; Paterson, I. Chem. Rev. 2005, 105, 4237.

(14) Morris, J. C.; Phillips, A. J. Nat. Prod. Rep. 2010, 27, 1186.

(15) Tang, S. A.; Deng, Z. W.; Li, J.; Fu, H. Z.; Pei, Y. H.; Zhang, S.;
Lin, W. H. Chin. Chem. Lett. 2005, 16, 353.

(16) Lv, F.; Deng, Z. W.; Li, J.; Fu, H. Z.; van Soest, R. W. M.; Proksch, P.; Lin, W. H. J. Nat Prod. 2004, 67, 2033.

(17) Lagunin, A.; Stepanchikova, A.; Filimonov, D.; Poroikov, V. Bioinformatics 2000, 16, 747.

(18) Filimonov, D. A.; Poroikov, V. V. Bioactive Compound Design: Possibilities for Industrial Use, 1st ed.; BIOS Scientific Publishers, Oxford (UK) BIOS Scientific Publishers: Oxford, UK, 1996; pp 47-56.

(19) ChemOffice Ultra 2005 (http://www.cambridgesoft.com/); Cambridge Soft Inc.: Cambridge, MA, 2005.

(20) SYBYL 6.91 (http://www.tripos.com); Tripos Inc.: St, Louis, MO, 2006.

(21) Lagunin, A.; Filimonov, D. A.; Poroikov, V. V. Cur. Phar. Des. 2010, 16, 1703.

(22) Ewing, T. J. A.; Makino, S.; Skillman, A. G.; Kuntz, I. D. J. Comput. Aid. Mol. Des. 2001, 15, 411.

(23) Lape, M.; Elam, C.; Paula, S. Biophys. Chem. 2010, 50, 88.

(24) Cross, J. B.; Thompson, D. C.; Rai, B. K.; Baber, J. C.; Fan, K. Y.; Hu, Y.; Humblet, C. J. Chem. Inf. Model. 2009, 49, 1455.

(25) McGaughey, G. B.; Sheridan, R. P.; Bayly, C. I.; Culberson, J. C.; Kreatsoulas, C.; Lindsley, S.; Maiorov, V.; Truchon, J. F.; Cornell, W. D. J. Chem. Inf. Model. 2007, 47, 1504.

(26) DesJarlais, R. L.; Gibbs, A. C.; Mohan, V.; Jaeger, E. P. J. Med. Chem. 2005, 48, 962.

(27) Perola, E.; Walters, W. P.; Charifson, P. S. Proteins 2004, 56, 235.

(28) Goodsell, D. S.; Morris, G. M.; Olson, A. J. J. Mol. Recognit. 1996, 9,1

(29) Morris, G. M.; Goodsell, D. S.; Halliday, R. S.; Huey, R.; Hart, W. E.; Belew, R. K.; Olson, A. J. J. Comput. Chem. 1998, 19, 1639. 